\title{
From fistula to disability. Severe infection due to anal fistula treated with NPWT - case report
}

\author{
Mateusz Szyntor, Radosław Wojciechowski, Magdalena Surudo
}

\section{CASE REPORT}

\begin{abstract}
Negative pressure wound therapy (NPWT) is increasingly used to facilitate wound healing. There are various wound types - primarily closed or open, clean or infected, superficial or deep-penetrating, open abdomen, enteroatmospheric fistulae, burns, etc. and almost each of these might be healed with the help of NPWT. It seems to be the most beneficial in hard-to-heal wounds. Using sub-atmospheric pressure, one can significantly accelerate the separating of necrotized tissues, stop inflammation, promote granulation, and drain the difficult wounds effectively. Typically, an optimum pressure range of -80 to $-125 \mathrm{mmHg}$ is administered. Few contraindications for the use of NPWT are challenged by new reports showing benefits in situations where NPWT was previously forbidden, (i.e. malignancy or bleeding in the wound), increasing the quality of life and avoiding wound infections. There is a growing body of data for new methods of treating anal fistula in the literature, but there are no data for elective treatment with NPWT. Attempts to develop NPWT protocols are problematic due to the diversity of cases and the frequent need for a personalized therapeutic approach.

This paper presents a 56-year-old male patient suffering from severe, purulent inflammation of groin, inguinal, anal and left gluteal sites; septic, malnourished, depressed, in continuously worsening general status since 3 years. Computed tomography and colonoscopy did not reveal pathology except for purulent inflammation. At the beginning, the patient was treated symptomatically with antibiotics, colostomy and surgical excision facilitated with NPWT. Anal fistulae were found after a large tisue excision. A medium-thick skin transplantation was performed and well accepted in over $90 \%$. This case presents a 103-day-long hospitalization with numerous complications. Finally, the wound healed completely and the patient was restored to his full physical abilities and his quality of life benefited greatly.

NPWT used in this case allowed for inflammation control, extensive wound healing and closing the anal fistula with no recurrence.
\end{abstract} apy

Keywords-anal fistula, VAC, negative pressure wound ther-

\section{INTRODUCTION}

A NAL fistula is a tract from the rectum or anus, with an internal opening that communicates with the skin around the anus at the external opening $!^{10}$ Latest literature

Manuscript received 17.03.2019; revised 04.04.2019. This work did not receive any financial support. Authors declare no conflict of interest.

Author affiliations: Independent public medical center Medicam in the memory of Witold and Julia Starkiewicz, address: ul. Niechorska 27, 72300 Gryfice, (MS, RW, MS)

*Correspondence to: Mateusz Szyntor: maq90@wp.pl reports an incidence as high as $21 / 100,000$. Anal fistulae occur most frequently between 30 and 50 years of age and are 2-6 times more prevalent in males than females. ${ }^{2}$ In 1976, Parks et al ${ }^{[3]}$ classified anal fistulae into four types by the tract course: extrasphincteric, suprasphincteric, transsphincteric and intersphincteric; further classifications added the submucosal type, which also was diagnosed in the described case. Since transrectal ultrasound (TRUS) and magnetic resonance imaging (MRI) became more available, the authors of newer papers have used it to classify fistulae into 5 grades $\cdot\left[\frac{[3]}{[5}\right.$ Symptoms include pain, difficulty sitting and purulent or bloody discharge when perianal abscesses are present. Patients may also have systemic sepsis. ${ }^{6}$ There are many therapeutic options for anal fistula. However, fistulotomy is the mainstay of treatment. Elective treatment is recommended, when active inflammation or abscesses are not present. ${ }^{7}[8$

Negative pressure wound therapy (NPWT) is used in all wound types and is especially useful when a hard-toheal wound is to be treated. Its benefits include draining exudate, contracting the wound edges, stimulating angiogenesis, reducing tissue edema, stimulating the formation of granulation tissue, and creating a moist environment. By altering the blood flow in the wound edges and stimulating the wound bed, NPWT improves drug penetration ${ }^{9[10}$ Using sub-atmospheric pressure accelerates the separating of ischemic tissues. Typically, an optimum pressure range of -80 to $-125 \mathrm{mmHg}$ is administered, but in the case described we decided to used $-200 \mathrm{mmHg}$ to reduce superficial bleeding and to keep the dressing tight.

The PubMed and Cochrane databases showed no results for a search of 'anal fistula' with 'NPWT'. There are reports on enteroatmospheric fistulae being treated with NPWT, which are a valuable source of knowledge, but the conclusions cannot be extrapolated entirely to anal fistulae. This paper presents a severe groin and perianal infection treated with a large excision and skin transplantation preceded with NPWT. Anal fistulae were found to be the cause relatively late during the therapy process.

\section{CASE REPort}

A 56-year-old male patient with no other comorbidities was admitted to a surgical ward due to a massive, non-healing buttock and perineum inflammation. The first symptoms had 

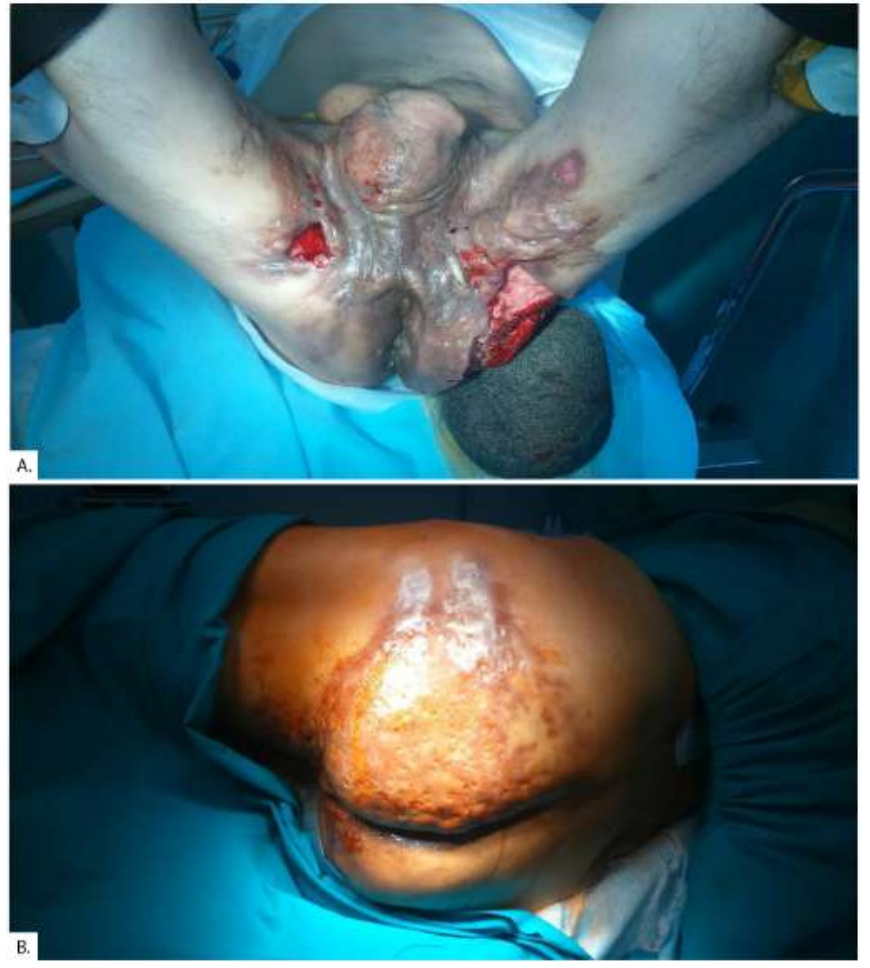

Figure 1. Status at admission: proper examination was possible under anaesthesia. Extensive soft tissue phlegmon in groin, scrotum, both inguinal, perianal and left gluteal site

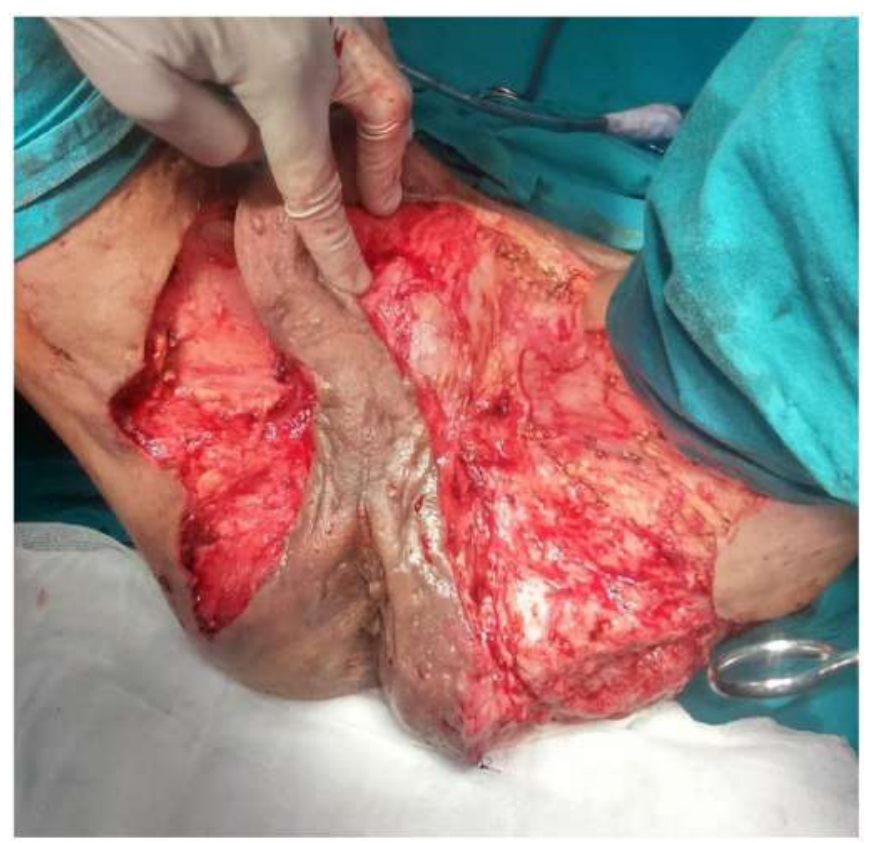

Figure 2. Subtotal excision of phlegmon tissues with margin. The perianal site was saved to keep tightness with NPWT dressing using adhesive gel patches. The anus was still excreting.
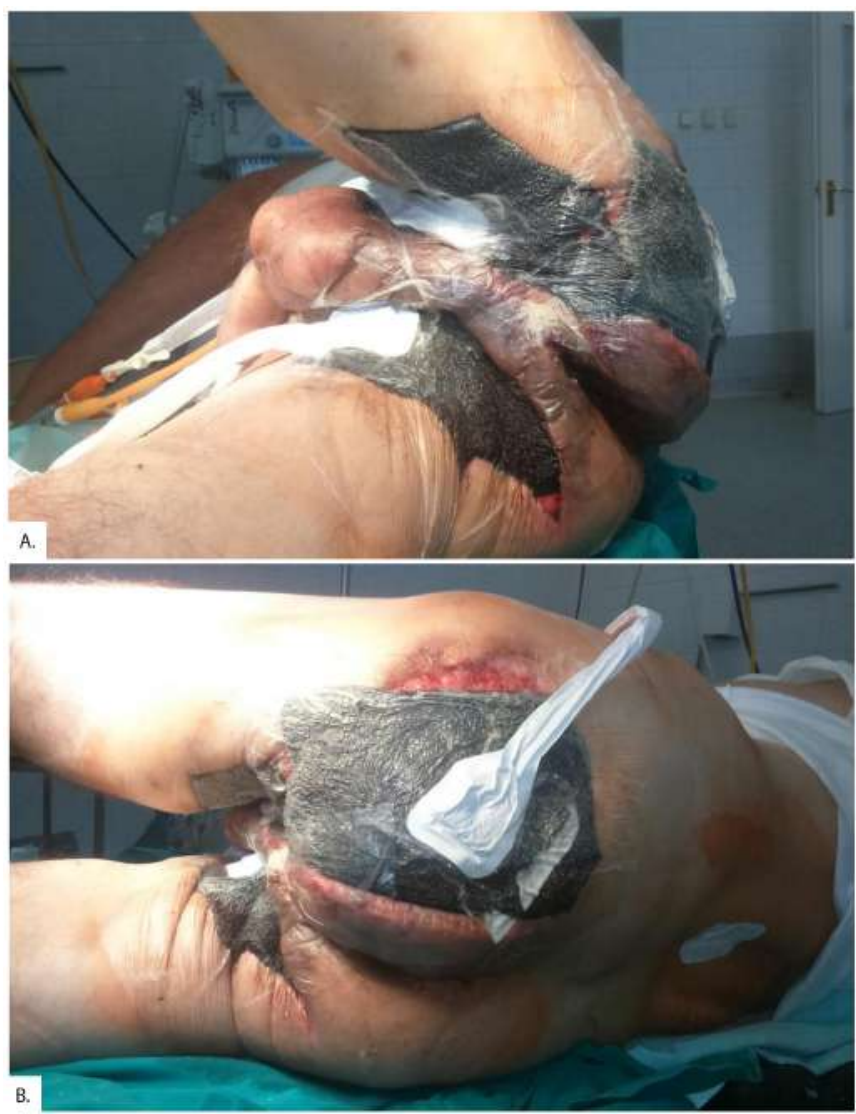

Figure 3. NPWT dressing. Two separated areas were connected with one processor by 3 soft ports. Adhesive gel patches applied perianally.

appeared 3 years previously as a small abscess on the scrotum base spreading superficially to both inguinal, anal and left gluteal sites. At this time, the patient had been treated in an out-patient clinic as well as hospitalized on infectious disease and dermatology wards; the patient did not deliver any past medical history reports. He had undergone multiple antimicrobial therapies combined with dressings and locallyacting antiseptic agents. There had been no satisfactory clinical improvement, and the disease progressed as his general status had gradually worsened. He presented with chronic fever, lost approximately $20 \mathrm{~kg}$ of body weight and had to cease his academic job due to the inability to sit and continued worsening of his general status.

On admission, the following were noted: bed-ridden, lower limb contractures, fever $>38^{\circ} \mathrm{C}$, pulse rate of approximately $110 / \mathrm{min}$, BMI $20.45 \mathrm{~kg} / \mathrm{m}^{2}$ (decreased from 26,3 during last 12 months; height $185 \mathrm{~cm}$, weight $70 \mathrm{~kg}$ ). Locally - extensive soft tissue phlegmon in the groin, scrotum, both inguinal sites, left gluteal site and perianally (Fig. 1), with voluminous purulent leak. In computer tomography and colonoscopy, no signs of anal fistula or any other pathologies were noted. In the other body areas, the skin was healthy. The patient had been examined by urologists on several occasions because of primary purulent fistula on the scrotum. Urological disease was excluded.

The patient was qualified for surgery. In the first step, the skin covering the left buttock was excised deeply to the 


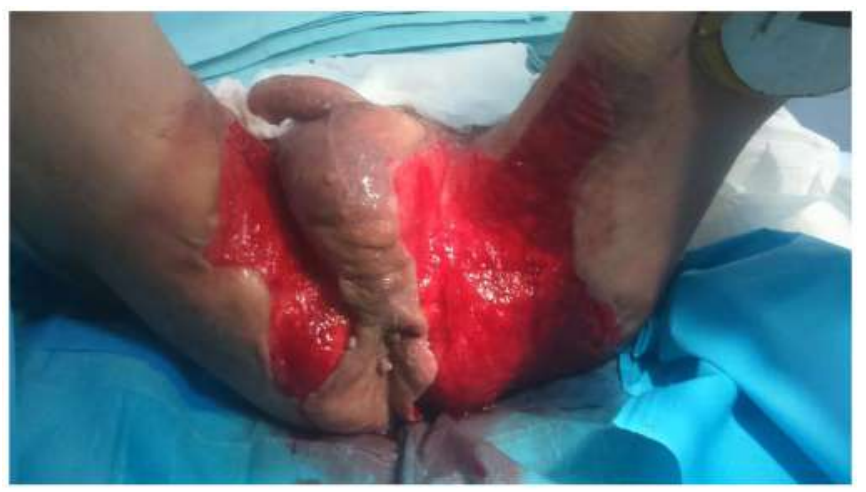

Figure 4. NPWT finished after 6 dressing changes. The wound was infected and covered with advanced biofilm, while there were positive blood samples with $S$. epidermidis and $C$. tropicalis - high risk of skin transplant rejection, systemic and local antimicrobial therapy was needed.

muscular fascia and an NPWT dressing was applied (pressure $-125 \mathrm{mmHg}$, continuous mode). After 5 days, tissues from the groin, scrotum and inguinal sites were excised with the margin (Fig. 2). The perianal site was saved to maintain tightness with NPWT close to the excreting anus. A subatmospheric dressing was placed on the whole wound with 3 soft ports (Fig. 3). Tightness was achieved using adhesive gel patches.

During dressing changes over the next 5 days, a significant improvement was observed. Local inflammation was reduced and the wound was granulating. In accordance with the presumed long starving period, laparoscopic sigmoid colostomy was performed. Oral feeding was restored. Without natural defecation, it became possible to excise perianal tissues radically and large abscesses in the mesorectum were emptied. Furthermore, filamentous transsphincteric and submucosal fistulae without internal aperture were found, which had previously been imperceptible. Fistulae were marsupialised. All spaces and fistulae tracts were filled with NPWT sponge and after 6 dressing changes (27 days), the negative pressure therapy was finished (Fig. 4). The main difficulty was a recurrent decompression of the dressing. Because of the pain, dressings were changed under anaesthesia. There were attempts to recover the tightness by adding more stoma paste, then drapes, and increasing the negative pressure $(-125 \mathrm{mmHg}$ was further decreased to even $-200 \mathrm{mmHg}$, continuous mode) and this was successful. The right half of the wound was sealed in one position and it was relatively easy. The left, larger half of the wound forced changing the patient's position from right-sided to gynaecological and back during the procedure. Leaking serous and bloody exudate made it difficult to keep the surrounding skin dry for applying the drape and the procedure needed to be performed very quickly.

The preparation of the wound for skin transplantation was impeded by infection. Dressings with Bacitracin, Neomycin, Povidone-iodine (transient hypersensitivity to iodine) and liquid paraffin were changed every 2-3 days. Silver-containing dressings had a poor clinical effect: biofilm and infection returned. Finally, satisfactory wound cleanliness was achieved

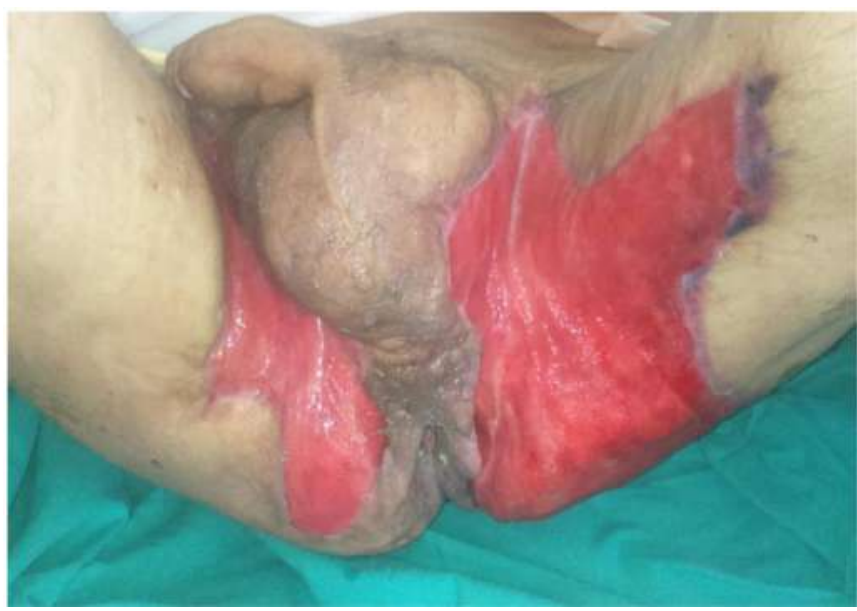

Figure 5. Satisfactory cleanliness before skin transplantation.

(Fig. 5). On the 62nd day of treatment a medium-thick skin transplantation on the buttock was performed, and on the 84th day, this was done on the whole groin area. It was accepted in over $90 \%$ and the whole wound and healed. No problems with the healing donor sites were observed. During hospitalization, the patient underwent the anaesthesia procedure for 32 times and the dressings were applied in opioid analgesia only.

Patient was discharged after a 103-day-long stay (Fig. 6) in a good general condition, walking supported with crutches, without contractures, eating a full oral diet fortified with oral nutritional supplementation, educated on how to change dressings. Good complience to the therapy was observed during weekly out-patient controls, which resulted in immediate restoration of previous mental and physical abilities, including sexual activities. Regular controls were discontinued after 5 months (Fig. 7). Pathological examination of a sample taken from the wound bed during follow up revealed severe inflammation features. There were no symptoms of anal fistula during the follow-up. The patient stays in contact with surgical and proctological clinics and awaits stoma closure.

Important parts of the treatment were early admission and aggressive nutritional therapy. Immediately on admission and before surgery the patient qualified for complete intravenous nutrition, because of two reasons: the first was to keep the wound clean and latter to turn a severe catabolism to anabolism. Due to chronic general inflammation and periodically worse renal function, the tolerance of intravenous nutrition was limited. Oral feeding was restored after performing colostomy, which allowed the intravenous dosage to be decreased. Delivery of whole caloric and protein needs became possible after combining oral with intravenous nutrition. Intravenous nutrition was finished after limiting general inflammation and protein loss through the wound. As a bed ridden patient, he was administered an enhanced oral diet $(5$ meals) fortified with enteral nutrition (commercial product Cubitan, Nutricia) 1-2 times a day and $1970 \mathrm{ml}$ of parenteral nutrition (commercial product SmofKabiven, Fresenius Kobi), which equals 4,663 kcal daily. During his whole stay, the patient received almost 140,000 kcal 


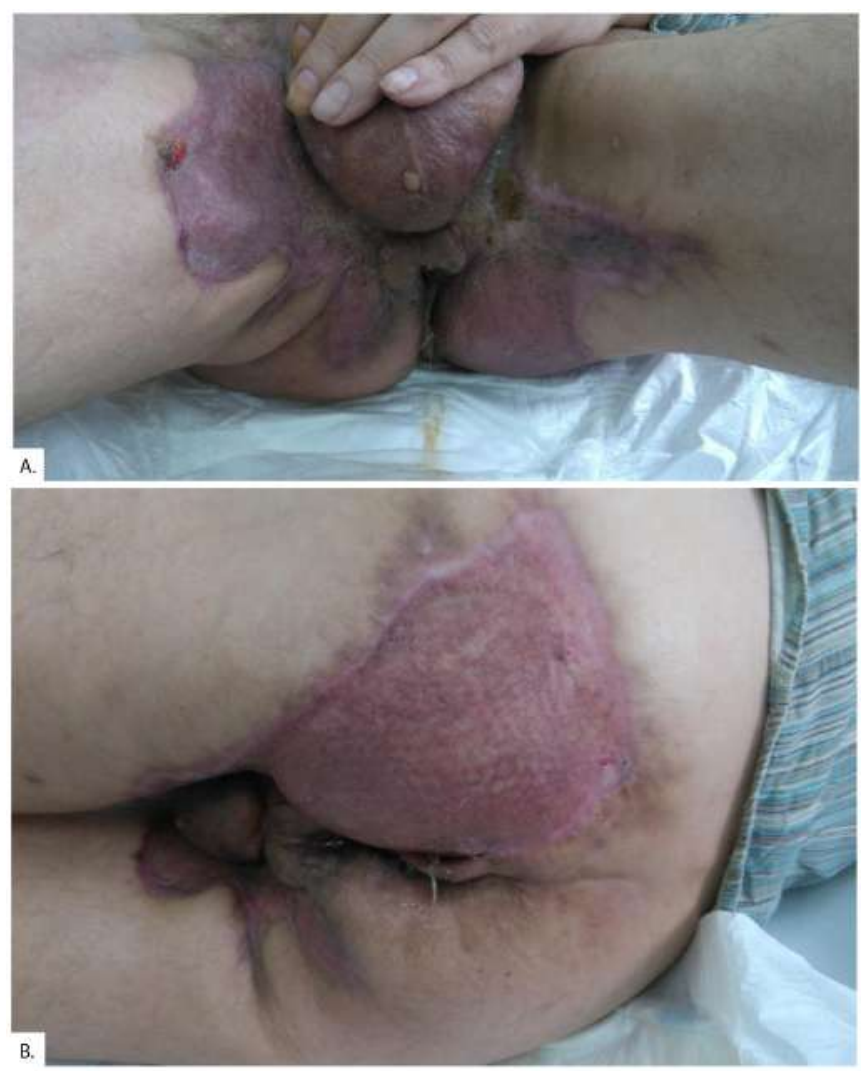

Figure 6. Discharge status. Medium-thickness skin transplant accepted in $>90 \%$, partly healed by granulation and epithelialization.

intravenously — assuming a mean of $7000 \mathrm{kcal}$ per $1 \mathrm{~kg}$ of body weight. The patient avoided $20 \mathrm{~kg}$ weight loss. His body mass index on discharge was similar to that on admission, but with no edema and after large excision. The patient had gained over $30 \mathrm{~kg}$ body mass by the 5 th-month follow-up.

Such a long and multimodal treatment had varied complications. In addition to difficulties with dressing, there was a problem with stoma. On the 3rd day after formation, it necrotized because of a too narrow fascia aperture. There was no need for relaparoscopy and the problem was solved by widening the aperture from the outside and shortening the colon by a few centimetres. Central venous ports for intravenous nutrition entailed more difficulties. There were recurrent sepses with $S$. epidermidis and $C$. tropicalis treated according to antibiograms with linezolid $0.6 \mathrm{~g} / 10$ days i.v. twice daily and fluconazole $0.4 \mathrm{~g} / 21$ days i.v. once daily. At the end of intravenous nutritional therapy, the right carotid, subclavian and axillary veins developed thrombophlebitis, which was treated successfully with enoxaparin $60 \mathrm{mg}$ twice a day for 3 months.

\section{DISCUSSION}

The use of NPWT after a large skin excision seemed the only possibility to stop or merely slow down the natural progression of the patient's disease. The clinical view of the affected skin was unquestionable in need of surgical intervention. Having experience with NPWT, we administered it on the extensive wound. We started with the standard protocol

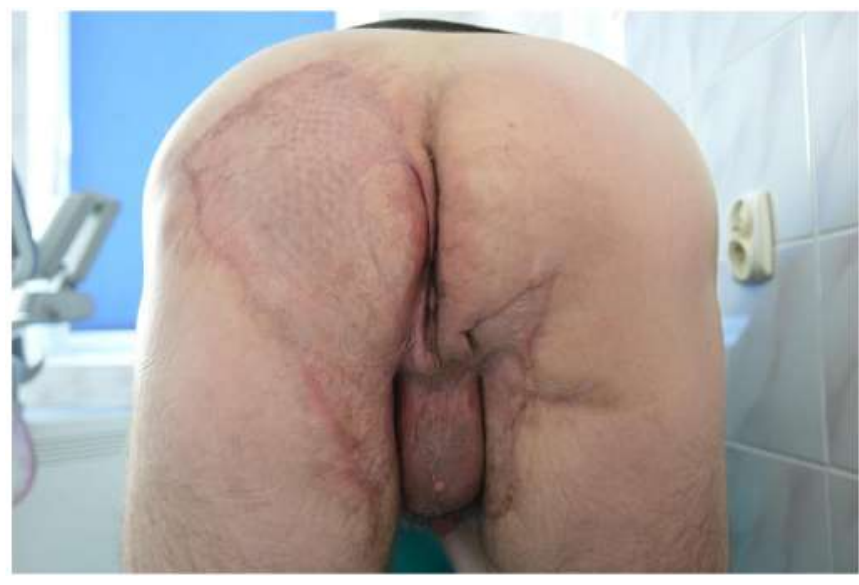

Figure 7. Follow-up after 5 months. Achieved skin elasticity with no contractures, full mobility of lower limbs with no pain. The regular outpatient control was finished.

of $-125 \mathrm{mmHg}$, continuous mode. The intermittent mode was useless because it caused an air leak. It was necessary to take a risk and increase the pressure to $-200 \mathrm{mmHg}$ near to the rectum (without unsheathed mucosa) for the purpose of keeping air-tightness. This appeared as effective therapy, which also decreased the exudate, as describe in the literature 11 Unfortunately, it was associated with severe pain and forced morphine use. When we found two simple anal fistulae without internal openings, we decided to fill them with sponge simultaneously, which resulted in proper healing of the tract without any recurrence.

Forming colostomy has recently became controversial; it seems to be an overtreatment in the face of modern management ${ }^{[12}$ The final effect which undoubtedly increased patient's quality of life suggests that NPWT might be a highly appropriate treatment option in very advanced cases. CT, colonoscopy and final pathological examination did not confirm the cause of fistulae, thus it seems that the proper diagnosis was spontaneous anal fistula spreading from anus, although there is no answer to the question why it spread so much in the adult patient without risk factors. We did not perform MRI because of the need for further anaesthesia, and the outcome of this diagnostic procedure would not change the therapy regimen.

Negative pressure wound therapy (NPWT) is especially beneficial for hard-to-heal wounds. 13 There are papers where its benefits are challenged. There are also reviews of primarily clean or potentially infected wounds which compared incisional NPWT to standard therapy and no significant differences between groups were shown.14 15 The case presented in this paper cannot be compared to others as we did not find any similar reports.

Due to the lack of data on healing anal fistula with negative pressure, we tried to compare our case with sitesimilar wounds. A study of pilonidal disease showed the benefits of incisional NPWT ${ }^{16}$ Moreoiver, papers discussing enteroatmospheric fistulae present the benefits of such an approach 17. 18 Using multiple dressings seems to be expensive, but Hampton in his cohort case study gives reasons for cost- 
effectiveness of such an approach 19

\section{CONCLUSION}

Negative pressure wound therapy applied to treat hard-toheal wounds significantly accelerates the recovery process. Because of the diversity of cases, a procedure protocol is difficult to develop and each case needs personalized approach. Applying NPWT in severe cases of anal fistula should be considered and requires further studies.

\section{REFERENCES}

[1] C. Marks and J. K. Ritchie, "Anal fistulas at st mark's hospital," British Journal of Surgery, vol. 64, no. 2, pp. 84-91, 1977.

[2] T. Yamana, "Japanese practice guidelines for anal disorders ii. anal fistula," Journal of the Anus, Rectum and Colon, vol. 2, no. 3, pp. 103-109, 2018.

[3] A. Parks, P. H. Gordon, and J. Hardcastle, "A classification of fistulain-ano," British Journal of Surgery, vol. 63, no. 1, pp. 1-12, 1976.

[4] J. Morris, J. A. Spencer, and N. S. Ambrose, "Mr imaging classification of perianal fistulas and its implications for patient management," Radiographics, vol. 20, no. 3, pp. 623-635, 2000.

[5] P. Garg, "Comparing existing classifications of fistula-in-ano in 440 operated patients: Is it time for a new classification? a retrospective cohort study," International Journal of Surgery, vol. 42, pp. 34-40, 2017.

[6] H. Owen, G. Buchanan, A. Schizas, R. Cohen, and A. Williams, "Quality of life with anal fistula," The Annals of The Royal College of Surgeons of England, vol. 98, no. 5, pp. 334-338, 2016.

[7] P. Shouler, R. Grimley, M. Keighley, and J. Alexander-Williams, "Fistula-in-ano is usually simple to manage surgically," International journal of colorectal disease, vol. 1, no. 2, pp. 113-115, 1986.

[8] E. Limura and P. Giordano, "Modern management of anal fistula," World Journal of Gastroenterology: WJG, vol. 21, no. 1, p. 12, 2015.

[9] T. Li, G. Wang, P. Yin, Z. Li, L. Zhang, J. Liu, M. Li, L. Zhang, L. Han, and P. Tang, "Effect of negative pressure on growth, secretion and biofilm formation of staphylococcus aureus," Antonie van Leeuwenhoek, vol. 108, no. 4, pp. 907-917, 2015.
[10] C. Huang, T. Leavitt, L. R. Bayer, and D. P. Orgill, "Effect of negative pressure wound therapy on wound healing." Current problems in surgery, vol. 51, no. 7, pp. 301-331, 2014.

[11] J. Białecki, P. Pyda, A. Kołodziejska, A. Rybak, and S. Sowier, "Applying npwt to bleeding open wounds after forefoot amputation in diabetic foot patients-a case report," Negative Pressure Wound Therapy Journal, vol. 5, no. 4, pp. 5-8, 2018 .

[12] E. Limura and P. Giordano, "Modern management of anal fistula," World Journal of Gastroenterology: WJG, vol. 21, no. 1, p. 12, 2015.

[13] R. Frazee, A. Manning, S. Abernathy, C. Isbell, T. Isbell, S. Kurek, J. Regner, R. Smith, and H. Papaconstantinou, "Open vs closed negative pressure wound therapy for contaminated and dirty surgical wounds: a prospective randomized comparison," Journal of the American College of Surgeons, vol. 226, no. 4, pp. 507-512, 2018.

[14] P. Shen, A. U. Blackham, S. Lewis, C. J. Clark, R. Howerton, H. D. Mogal, R. M. Dodson, G. B. Russell, and E. A. Levine, "Phase ii randomized trial of negative-pressure wound therapy to decrease surgical site infection in patients undergoing laparotomy for gastrointestinal, pancreatic, and peritoneal surface malignancies," Journal of the American College of Surgeons, vol. 224, no. 4, pp. 726-737, 2017.

[15] V. Zotes, J. M. Mier, and G. Cortes, "P-187negative pressure wound therapy in a potentially infected wound after empyema surgery," Interactive cardiovascular and thoracic surgery, vol. 21, no. suppl_1, pp. S51-S51, 2015.

[16] E. Bianchi, J. Lei, T. Adegboyega, S. S. Shih, M. Berrones, S. Purdy, and D. E. Rivadeneira, "Negative pressure wound therapy is beneficial in the treatment of pilonidal disease," Journal of the American College of Surgeons, vol. 227, no. 4, pp. e109-e110, 2018.

[17] C. Terzi, T. Egeli, A. E. Canda, and N. C. Arslan, "Management of enteroatmospheric fistulae," International wound journal, vol. 11, no. s1, pp. 17-21, 2014.

[18] A. Bobkiewicz, D. Walczak, S. Smoliński, T. Kasprzyk, A. Studniarek, M. Borejsza-Wysocki, A. Ratajczak, R. Marciniak, M. Drews, and T. Banasiewicz, "Management of enteroatmospheric fistula with negative pressure wound therapy in open abdomen treatment: a multicentre observational study," International wound journal, vol. 14, no. 1, pp. 255-264, 2017.

[19] J. Hampton, "Providing cost-effective treatment of hard-to-heal wounds in the community through use of npwt," British journal of community nursing, vol. 20, no. Sup6, pp. S14-S20, 2015. 